\title{
Erratum zu: Eberhard Siebert, Heinrich von Kleist
}

Klaus Müller-Salget

\section{Erratum zu: \\ Kapitel „Eberhard Siebert, Heinrich von Kleist“ in: A. Allerkamp et al. (Hrsg.), Kleist-Jahrbuch 2021, Kleist-Jahrbuch https://doi.org/10.1007/978-3-662-64174-3_15}

Die Online-Version dieses Kapitels wurde versehentlich mit einem falschen Autorennamen „Klaus cc“ veröffentlicht. Der Name des Autors wurde korrigiert in: Klaus Müller-Salget. 